\title{
Genotyping of Brucella species using clade specific SNPs
}

Jeffrey T Foster ${ }^{1 *}$, Lance B Price ${ }^{2}$, Stephen M Beckstrom-Sternberg ${ }^{1,3}$, Talima Pearson ${ }^{1}$, William D Brown ${ }^{1}$, Danika M Kiesling ${ }^{1}$, Christina A Allen ${ }^{1}$, Cindy M Liu ${ }^{1,2}$, James Beckstrom-Sternberg ${ }^{3}$, Frank F Roberto ${ }^{4}$ and Paul Keim ${ }^{1,2}$

\begin{abstract}
Background: Brucellosis is a worldwide disease of mammals caused by Alphaproteobacteria in the genus Brucella. The genus is genetically monomorphic, requiring extensive genotyping to differentiate isolates. We utilized two different genotyping strategies to characterize isolates. First, we developed a microarray-based assay based on 1000 single nucleotide polymorphisms (SNPs) that were identified from whole genome comparisons of two B. abortus isolates, one B. melitensis, and one B. suis. We then genotyped a diverse collection of 85 Brucella strains at these SNP loci and generated a phylogenetic tree of relationships. Second, we developed a selective primer-extension assay system using capillary electrophoresis that targeted 17 high value SNPs across 8 major branches of the phylogeny and determined their genotypes in a large collection $(n=340)$ of diverse isolates.

Results: Our 1000 SNP microarray readily distinguished B. abortus, B. melitensis, and B. suis, differentiating B. melitensis and B. suis into two clades each. Brucella abortus was divided into four major clades. Our capillary-based SNP genotyping confirmed all major branches from the microarray assay and assigned all samples to defined lineages. Isolates from these lineages and closely related isolates, among the most commonly encountered lineages worldwide, can now be quickly and easily identified and genetically characterized.

Conclusions: We have identified clade-specific SNPs in Brucella that can be used for rapid assignment into major groups below the species level in the three main Brucella species. Our assays represent SNP genotyping approaches that can reliably determine the evolutionary relationships of bacterial isolates without the need for whole genome sequencing of all isolates.
\end{abstract}

\section{Background}

Brucella are Gram-negative bacteria and the causative agent of brucellosis in domesticated animals, wildlife, and humans. Although the bacteria exhibit relatively strong host preference, separating the various Brucella species has proven extremely difficult due to minimal genetic differentiation [1]. Based on DNA-DNA hybridization, brucellae have previously been proposed to comprise a single species, with a series of biovars [2]. However, phylogenetic approaches explicitly incorporating host preference and virulence have upheld the six classical Brucella species: B. abortus (bovine), B. melitensis (caprine and ovine), B. suis (porcine), B. canis

\footnotetext{
* Correspondence: jeff.foster@nau.edu

${ }^{1}$ Center for Microbial Genetics and Genomics, Northern Arizona University, Flagstaff, AZ 86011-4073, USA

Full list of author information is available at the end of the article
}

(canine), B. neotomae (desert woodrat), and B. ovis (ovine) [3-5]. Several new species have been recently described, including at least two species in marine mammals (B. ceti in dolphins, porpoises, and whales and $B$. pinnipedialis in seals) [6] and an additional species $B$. microti in the common vole (Microtus arvalis) [7]. Other Brucella species undoubtedly exist within known and novel hosts [8-11].

The limited genetic differentiation and conservation within Brucella genomes has made genotyping a challenge. A promising approach that is capable of being incorporated into high-throughput assays is the use of single nucleotide polymorphisms (SNPs). Comparisons of Brucella genomes have revealed hundreds of SNPs that distinguish various strains [12-14]. Although the era of Next-Generation sequencing [reviewed in 15] is rapidly increasing available data for microbial genomic 
comparisons, full genome sequencing is currently not cost effective for genotyping large numbers of isolates and requires intensive bioinformatic efforts. Furthermore, in low diversity organisms such as Brucella only a small fraction of the nucleotides are polymorphic, suggesting that once rare polymorphisms are discovered, methods other than whole genome sequencing are more efficient for most purposes.

Molecular Inversion Probe (MIP) assays are an efficient and relatively inexpensive method of interrogating thousands of SNPs in large numbers of samples [16]. Although typically applied to research on human disease, the MIP assay can be readily applied to genotype SNPs in bacterial genomes. We compared four genomes from B. abortus, B. melitensis, and B. suis to discover SNPs. We created a MIP assay to genotype 85 diverse samples and to discover canonical SNPs [17] that define Brucella species, strains, or isolates. We then created SNPspecific assays that use a Capillary electrophoresis Universal-tailed Mismatch Amplification mutation assay (CUMA) approach for major branch points in the phylogeny and screened them against a large and diverse collection of isolates $(n=340)$. Finally, we compared these results to 28 Brucella whole genomes in silico to place our genotyping into context with all major biovars and isolates.

\section{Results}

A total of 833 MIP probes consistently amplified their target sites across 85 samples. Among these probes, 777 identified truly polymorphic sites. This dataset contained only $4 \%$ missing data (2,636 no calls in 66,045 SNPs), where no SNP was determined at a particular locus for a sample. Comparisons to SNPs from 28 whole genomes yielded 735 SNPs in our phylogenetic analysis (Figure 1), allowing for placement of these genomes within the MIP tree and in silico determination of their SNP alleles. The full MIP tree with all 777 loci and 85 samples, excluding the whole genomes in the comparisons, is also given (Additional file 1: Figure S1).

Our MIP assay distinguished B. abortus, B. melitensis, and B. suis; the three prominent Brucella species (Figure 1). A total of 524 SNP loci had complete allele calls (i.e. no missing data) across all 85 samples. The assay strongly differentiated B. melitensis and B. suis into two clades each. Within B. melitensis, at least 27 SNPs on branch $\mathrm{H}$ separate strain $16 \mathrm{M}$ and its related isolates in biovar 1 from isolate 63-9 and related isolates of biovars 2. Subsequent analyses (see below) group biovar 3 with biovar 2 isolates. Based on these data, the assay for branch $\mathrm{H}$ appears to be specific to $B$. melitensis biovar 1 . The two clades in B. suis, denoted by branches I and J, included all isolates of in the species except for biovar 5, which was distantly related to other members of this species. Some isolates from $B$. suis are more closely related to $B$. canis isolates (branch J) than to other $B$. suis isolates (branch I), indicating that $B$. suis is a paraphyletic species. Of the SNPs with complete genotyping data, at least 31 SNPs on branch I separate B. suis 1330 and related isolates from $B$. canis and related $B$. canis and $B$. suis isolates. However, no SNPs uniquely identified B. canis. Brucella abortus was even more differentiated, and can be divided into at least four distinct clades. Samples from B. abortus biovar 1, which contains the two SNP discovery strains, plus the type strain for biovar 2 (strain 86/8/59), make up the majority of samples and diversity within the $B$. abortus clade. All were found on branch $E$, which was further divided into branches A-D. Samples from the other B. abortus biovars are more distantly related and form distinct branches. As expected, the other species in the assay, including B. neotomae, B. ceti, B. pinnipedialis, and B. ovis were poorly distinguished from each other. Missing data for SNP loci caused the differences in branch lengths that are seen between Figure 1 and Figure S1.

CUMA assays verified the SNP alleles for all 85 of the samples run in the MIP assay. In addition, the 17 SNPs from the CUMA assays allowed for placement of a larger panel of 340 isolates within the MIP phylogeny (Additional file 2: Table S3). Nine of these SNPs were located on the same branch as another assay, or in one instance the same branch as two other assays, so did not provide any additional genotyping information. In the final tally there were assays for eight branches of the phylogeny, with assays specific to the following prominent isolates/clades and related isolates: B. abortus 2308, B. abortus $2308+\mathrm{S} 19$, B. melitensis $16 \mathrm{M}$, B. melitensis biovar 1 , and $B$. suis 1330 . From our diverse isolate collection we had the following distribution of calls for the branches, with the most derived call taking precedence over more ancestral calls: $\mathrm{A}=1, \mathrm{~B}=23, \mathrm{C}=8, \mathrm{D}=22$, $\mathrm{E}=7, \mathrm{~F}=0, \mathrm{G}=15, \mathrm{H}=91, \mathrm{I}=33, \mathrm{~J}=17$, no derived call (all isolates not in species B. abortus, B. melitensis, or $B$. suis $($ canis $)=25$, no call for any assay $=7$, ancestral within $B$. abortus $=12$, ancestral within $B$. melitensis $=68$, ancestral within $B$. suis $=11$.

\section{Discussion}

Our assays show clear distinctions within and among $B$. abortus, B. melitensis, and B. suis. Our CUMA assays targeted clade-specific SNPs that can be incorporated into most other genotyping assays such as TaqMan Realtime PCR for increased sensitivity $[18,19]$. We have identified several important targets that should prove useful for clinical, epidemiological, and forensic purposes. For example, the assays targeting branches A, D, and I are specific to isolates closely related to $B$. abortus 2308 and B. abortus 9-941, and B. suis 1330, 


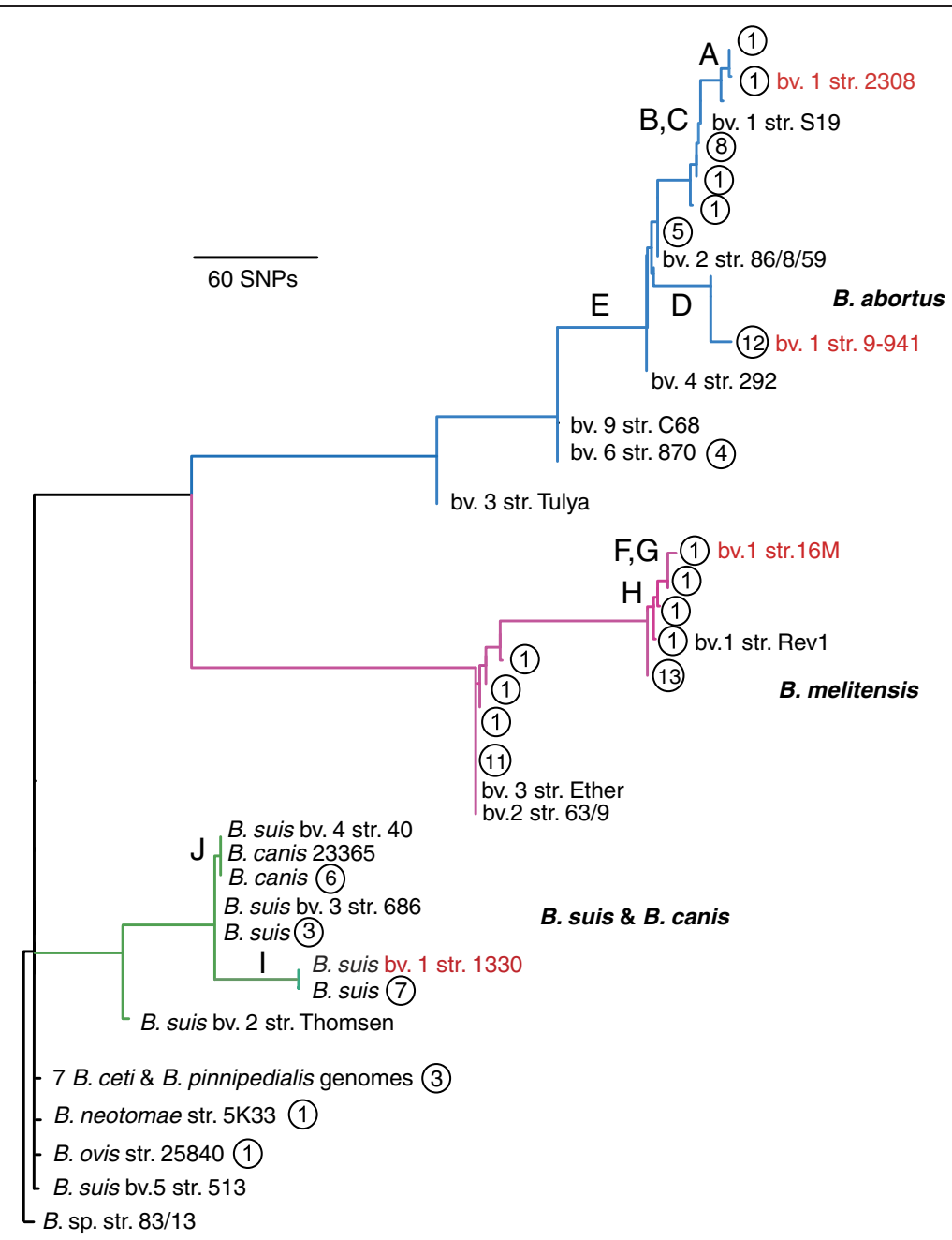

Figure 1 Brucella phylogeny based on comparison of 735 single nucleotide polymorphisms screened using Molecular Inversion Probes (MIP) in 85 samples and then compared to those SNPs in $\mathbf{2 8}$ whole genome sequences, which are the named isolates in the tree. Discovery genomes are indicated in red. Letters on branches refer to phylogenetic locations of CUMA assays developed in this work and genotyped against DNA from a diverse collection of 340 isolates. Circled numbers indicate the number of isolates with identical MIP genotypes (allelic profiles) at that branch location.

respectively. The assays for $\mathrm{F}$ and $\mathrm{G}$ target the same branch and identify $B$. melitensis $16 \mathrm{M}$ and closely related isolates. Isolates from $B$. abortus 2308 and 9941, B. suis 1330, and B. melitensis $16 \mathrm{M}$ are from common, genetically monomorphic clades of Brucella and the SNP assays developed here are a reliable and useful way of identifying these four common groups.

Branch E is particularly interesting in terms of Brucella taxonomy. The clade that this branch defines includes isolates from B. abortus biovars 1, 2, and 4. Potential issues with biovar and phylogenetic correspondence in B. abortus have been noted previously [20]. Upon closer evaluation of the whole genomes used in our analyses, the apparent paraphyly within $B$. abortus biovar 1 , since isolates from biovar 2 are within the biovar 1 clade, does not hold true when all the genomes are included. However, CUMA assays indicate that at least four isolates from other $B$. abortus biovars (3 of biovar 4,1 of biovar 2) fall onto the $\mathrm{B} / \mathrm{C}$ branch. This would suggest that either biovar 1 is paraphyletic or there have been issues with biovar determination.

SNP-based approaches also enable assessment of errors in genome sequences. Whole genome comparisons of the region associated with SNP10621, which were intended to target branch $J$ in $B$. suis/B. canis, also share a SNP allele with $B$. abortus 9-941. Taken at face value, this would suggest homoplasy at this locus. Yet, in our CUMA assays B. abortus 9-941 did not group with $B$. suis, likely indicating sequencing error.

Finding nucleotide polymorphisms that differentiate clades, species, or isolates is dependent on the genomes used for SNP discovery. In general, one will only find 
those SNPs that exist among the genomic samples used in the comparisons and novel SNPs will remain undiscovered [21]. This discovery bias can strongly affect taxonomic interpretation of results [22,23]. Although discovery bias is often less consequential for genotyping efforts, the effects of our choice of strains for SNP discovery are clearly apparent in our phylogenetic tree. The discovery strains are distinguished by their positions at terminal branches in the phylogeny. There is greater diversity observed in $B$. abortus simply because two strains were part of the discovery panel. Furthermore, although isolates on a branch will be grouped by the SNPs they share (or do not share), additional structure exists in the "true" phylogeny that is not apparent in the genotype tree. Branch lengths are also highly affected by the SNP discovery process. Species that are basal within this phylogeny, such as B. ceti, B. pinnipedialis, B. ovis, and $B$. neotomae have short branch lengths merely because these genomes were not part of SNP discovery. It must also be noted that B. suis biovar 5 is part of this basal group. SNPs that should group it with the rest of the $B$. suis clade were not present in our MIP assay, which is not surprising since this branch is extremely short, even with whole genome analysis [JTF unpubl. data, [24]. We did not observe differentiation of these and the other Brucella species, nor did we expect it because genomes from these groups were not a part of SNP discovery.

Whole genome resequencing at the Broad Institute of MIT/Harvard recently generated genomes for over 100 additional Brucella strains and these genomes should provide a broad basis for future genotyping efforts, with canonical SNPs developed for each of the important isolates and clades. Future genotyping efforts should include SNPs from all of the recognized species and biovars. Comparative work using some of these genomes has already been fruitful, demonstrating the emergence of the marine Brucella from within the terrestrial Brucella and showing a methodology for whole genome analysis [24].

A trade-off exists in current genotyping efforts between throughput and genomic sampling. Does one aim for a maximum amount of potentially informative loci through approaches such as whole genome sequencing but having to sacrifice the number of isolates that can be evaluated? Or does one aim for more complete sampling of large numbers of isolates but with a limited set of loci using individual SNP assays such as CUMA? Of course the ultimate answer depends on your research interest or clinical application as well as the amount of resources at hand. MIP assays provide phylogenetic resolution for an intermediate number of samples and intermediate number of SNPs. Nonetheless, MIP assays, or any assays based on previously discovered SNPs, will always have their inference limited by the genomes used in SNP discovery [21]. MIP assays do however allow for a focus on resolving branches of specific interest. Data from these assays then allows for targeted down selection of loci so that focal branches and isolates on them can be thoroughly interrogated using individual SNP assays. Identifying canonical SNPs and verifying their ability to differentiate clades by screening large numbers of isolates is the essential part of genotyping [17]. Less important is the type of assay used for SNP differentiation because it is highly dependent on the numbers of SNPs and samples one wants to screen. The MIP and CUMA SNP screening techniques are just two of many methods that can be used for SNP genotyping in Brucella and other bacteria.

\section{Conclusions}

We developed and evaluated two different SNP-based genotyping systems for three well studied species of Brucella: B. abortus, B. melitensis, and B. suis. The first genotyping approach, using Molecular Inversion Probes, divided the species into its three respective groups and allowed for finer scale genetic resolution. Notably, this resolution occurred almost entirely within the lineages of the four strains that were used for SNP discovery: $B$. abortus 2308, B. abortus 9-941, B. melitensis $16 \mathrm{M}$, and $B$. suis 1330 . This is to be expected since the choice of genomes for SNP discovery has a pervasive effect on the phylogenetic patterns that can be determined. We followed the MIP assay with development of Capillary electrophoresis Universal-tailed Mismatch Amplification mutation assays that targeted major branch points in the MIP phylogeny. We then genotyped a large and diverse collection of isolates. The main result is the development of fine scale genotyping assays that target among the most important and widespread lineages of Brucella. Moreover, these and closely related isolates can be easily and quickly distinguished from all other Brucella isolates.

Despite the era of whole genome sequencing being upon us, SNP-based genotyping and other targeted assays will remain relevant. Sequencing technology is advancing rapidly and costs per genome are quickly diminishing such that whole genome genotyping is the future of phylogenetics, forensics, and diagnostics. In fact, whole genome genotyping will soon be cost competitive with most other genotyping strategies and will have the advantage of capturing nearly all of the genetic variation with no issues of discovery bias. Nonetheless, targeted assays will remain a viable option for such goals as rapidly and easily characterizing large strain collections, clinical samples, and samples containing only trace amounts of DNA. Concerted efforts must be made to incorporate data from earlier genotyping strategies into 
genomic databases so this wealth of genetic information is not lost in the rush to sequence everything.

\section{Methods}

\section{SNP selection}

SNPs were selected by comparisons of the four Brucella genomes that were available at the time of MIP development: B. melitensis $16 \mathrm{M}$ [25], B. suis 1330 [13], B. abortus 2308 [26] and B. abortus 9-941 [12]. SNPs from the whole genome sequences were discovered using an inhouse pipeline that performs pairwise comparisons of 200 base regions around each SNP using MUMMER [see [14]. Determining the quality of the putative SNPs is essential because only high quality sequence data should be used for developing genotyping analyses [27]. Quality measures included the number of bases between SNPs and the number of bases that are conserved on each side of a SNP within a specified region. To reduce the potential effects of sequencing error, we then incorporated sequencing quality scores from Phred values. We selected only those putative SNPs with quality scores $\geq 30$, average quality scores of SNP flanking regions (30 base pairs) $\geq 30$, and where each base in the flanking regions had a quality score $\geq 20$. Perl and Java scripts were then employed for additional alignments and to compile and summarize the data. Using this process, 1000 putative SNPs were selected for interrogation by the MIP chip. SNP locations and flanking regions of 40 bases on each side were sent to the manufacturer for assay design (Affymetrix, Santa Clara, CA).

\section{MIP primers and probes}

The MIP workflow is relatively straightforward: 1) SNPs are first discovered using comparisons of whole genomes or particular regions of interest within sequenced genomes; 2) a series of assays are created with primers targeting each SNP; 3) amplification products are generated in a single multiplexed PCR; 4) amplicons specific to each SNP for each sample are hybridized to a universal tag microarray; 5) each SNP is fluorescently labeled based on the corresponding nucleotide of the sample and is then visualized on the microarray.

Primers and probes were designed for a GeneChip Custom 5 K SNP Kit (Affymetrix), which is one of the available forms of the MIP assay. In this assay, all 1000 SNPs were assessed in a single multiplex reaction for each sample. Assays containing 3000 Francisella tularensis SNPs [28] and 1000 Burkholderia pseudomallei SNPs (Keim unpubl. data) were run concurrently on the same chip, which reduced the cost of the assays for each group. MIP technology involves a specific probe that binds to flanking sequence surrounding a SNP site. Due to the orientation of the oligonucleotide sequence, the probe anneals as an inverted loop and a single base gap is created at the SNP site. The base at the SNP site is then added in one of four reactions involving unlabeled nucleotides. After ligation and exonuclease steps, the probe released from the sequence is amplified with PCR using universal primers specific for a portion of all probes. Only those probes where the SNP base has been added are successfully amplified. For a full description of the MIP methodology, see Hardenbol et al. [16]. Typically, approximately $80 \%$ of the MIP probes that are designed pass quality control and assurance standards at Affymetrix. Consistent with this standard, 833 of the 1000 probes (83.3\%) amplified in our panel of 85 Brucella isolates for at least $80 \%$ of SNP alleles at a locus. Among these SNPs, 56 were monomorphic, leaving a final set of 777 phylogenetically informative loci. This dataset contained only $4 \%$ missing data, which were given an allele of $\mathrm{N}$ in phylogenetic analyses. To allow this dataset to be directly comparable to SNPs from whole genome analyses, we then did an in silico comparison of 28 whole genome sequences of Brucella from GenBank (Additional file 3: Table S1). Not all of the SNPs in the final set were present in all genomes or had likely duplication events so were removed from the analysis, resulting in 735 SNPs for phylogenetic analysis.

\section{DNA samples}

We ran 85 Brucella DNA samples on the MIP assay from a diverse isolate collection that included $B$. abortus (33), B. melitensis (30), B. suis (11), B. canis (6), B. neotomae (1), B. ovis (1), B. ceti (1), and B. pinnipedialis (2). The 85 samples tested are indicated (Additional file 4: Table S2). We focused our sampling on the first three species because SNP discovery had been conducted with the genomes of only these species and thus differentiation would be restricted primarily to these species $[21,22]$. Samples were analyzed at a range of concentrations, from $0.6-20 \mathrm{ng} / \mu \mathrm{l}$. Our larger panel of isolates $(n=340)$, used only in the CUMA assays (detailed below), is from a portion of our DNA collection, which came from a variety of sources (Additional file 4: Table S2). DNA was extracted using several different methods including chloroform, kit-based, and heat soak DNA extractions, although the extraction method was not always known for each sample. Isolates were largely recent, coming from sampling in the past 15 years. We note that the majority of samples came from the United States so this collection does not represent a truly global sampling.

\section{Phylogenetics and CUMA assays}

We created a matrix of SNP alleles for all SNP positions and formatted the data as one concatenated sequence for each sample. We analyzed this sequence in PAUP* using a heuristic search with the maximum parsimony 
Table 1 Capillary electrophoresis Universal-tailed Mismatch Amplification mutation assays for genotyping single nucleotide polymorphisms in Brucella isolates

\begin{tabular}{|c|c|c|c|c|c|c|c|c|}
\hline $\begin{array}{l}\text { SNP } \\
\text { assay }\end{array}$ & Allelic Primer1 & Allelic Primer2 & Consensus Primer & $\begin{array}{l}\text { Amplicon } \\
\text { Size }\end{array}$ & $\begin{array}{l}\text { Dye } \\
\text { Set }\end{array}$ & Allele & $\begin{array}{c}\text { SNP } \\
\text { position } \\
\text { in } 16 \mathrm{M}\end{array}$ & Branch \\
\hline 2366 & CACGGCCTATCTGCTGGGCT & CACGGCCTATCTGCTGGGAC & GAGCGTCGTGAAGTCGGTTAC & 151 & $\begin{array}{l}\text { NED/ } \\
\text { PET }\end{array}$ & $\mathrm{T} / \mathrm{C}$ & 1173417 & A \\
\hline 4748 & ACAGTCAGACAAGGACCGGA & AACAGTCAGACAAGGACCGAC & GTAACAAGAACACGGCCTTTACGC & 155 & $\begin{array}{l}\text { FAM/ } \\
\text { VIC }\end{array}$ & $C / A$ & 1744207 & B \\
\hline 1562 & CACGCAAAATAGCTAAATGAAATATAC & ACGCAAAATAGCTAAATGAAATATTG & GATGGCTTTCCGGGGCTATC & 189 & $\begin{array}{l}\text { NED/ } \\
\text { PET }\end{array}$ & $C / G$ & 1405281 & B \\
\hline 2922 & TAAAGACGGCGATTACCGAG & GTAAAGACGGCGATTACCGTA & GACAACGCCAACGGCATTCTT & $179-180$ & $\begin{array}{l}\text { NED/ } \\
\text { PET }\end{array}$ & $\mathrm{G} / \mathrm{A}$ & 413931 & C \\
\hline 991 & CAGTATGAAGCTTATTTTAAGCCA & GCAGTATGAAGCTTATTTAAGGCAG & GTATGCTCAAGCGCCAAGCTG & 195 & $\begin{array}{l}\text { FAM/ } \\
\text { VIC }\end{array}$ & $\mathrm{G} / \mathrm{A}$ & 1601481 & C \\
\hline 3740 & CGGAATACGAAAACTCACATTATAG & CGGAATACGAAAACTCACATTATTA & GCGGGGCCATAGGGAAATAC & 133 & $\begin{array}{l}\text { VIC/ } \\
\text { FAM }\end{array}$ & $G / A$ & 675905 & $\mathrm{D}$ \\
\hline 1344 & ACACGGTTGGAATTATCCACT & ACACGGTTGGAATTATCCATC & GACCGGCAAGCTTGAATCG & 171 & $\begin{array}{l}\text { FAM/ } \\
\text { VIC }\end{array}$ & $\mathrm{C} / \mathrm{T}$ & 1392400 & $\mathrm{D}$ \\
\hline 5754 & GCTGGAACATATAGAAAAGATCATAAAAG & GCTGGAACATATAGAAAAGATCATAAATA & GCAGCCTTCCAAGGAAAAGAACG & 117 & $\begin{array}{l}\text { VIC/ } \\
\text { FAM }\end{array}$ & $\mathrm{G} / \mathrm{A}$ & 1083478 & $\mathrm{E}$ \\
\hline 1522 & GGTGAACATTTCGCCATCAG & GGTGAACATTTCGCCATCTA & GTTCGATGAACCTCGTGGCATT & $123-124$ & $\begin{array}{l}\text { NED/ } \\
\text { PET }\end{array}$ & $G / A$ & 497534 & $\mathrm{E}$ \\
\hline 6214 & ATTGAATGATGAGCGATATTGTG & TGAATGATGAGCGATATTGCA & GAGCGCTTGTCGGAGGTTGTT & $110-112$ & $\begin{array}{l}\text { PET/ } \\
\text { NED }\end{array}$ & $A / G$ & 242224 & $\mathrm{~F}$ \\
\hline 2995 & CGAAACAGCTGAGAAGATCGAG & AAACAGCTGAGAAGATCGGC & GTTAGAAGCCTGGCCCGTTCTC & 101 & $\begin{array}{l}\text { VIC/ } \\
\text { FAM }\end{array}$ & $\mathrm{G} / \mathrm{C}$ & 478183 & G \\
\hline 8872 & GCATCGAACTCATTCTCGCT & GCATCGAACTCATTCTCGTC & GCGAAATCAAGGCCCCATTTG & 160 & $\begin{array}{l}\text { PET/ } \\
\text { NED }\end{array}$ & $\mathrm{C} / \mathrm{T}$ & 1170581 & G \\
\hline 1688 & CGATCTGCCAGTTGACGAGA & CGATCTGCCAGTTGACGATT & GTGCAACGCCTCACGCATAAT & 181 & $\begin{array}{l}\text { FAM/ } \\
\text { VIC }\end{array}$ & $\mathrm{T} / \mathrm{A}$ & 1348434 & $\mathrm{H}$ \\
\hline 5362 & ATTACCACGCACCGATGAGA & ATTACCACGCACCGATGAAG & GCATCCATGACGGCGTGAAAC & 240 & $\begin{array}{l}\text { FAM/ } \\
\text { VIC }\end{array}$ & $\mathrm{G} / \mathrm{A}$ & 507275 & । \\
\hline 8306 & CACGGTTGCATGGTTTGTATATA & ACGGTTGCATGGTTTGTATAAG & GAGCACCAAACCGGGTGATGT & 259 & $\begin{array}{l}\text { FAM/ } \\
\text { VIC }\end{array}$ & $\mathrm{G} / \mathrm{A}$ & 1876820 & । \\
\hline 10621 & TATGCAATTCGTGTCGCATG & TATGCAATTCGTGTCGCAGA & GTTTCAGGACTTTTGGGAACTGACC & 233 & $\begin{array}{l}\text { NED/ } \\
\text { PET }\end{array}$ & $\mathrm{G} / \mathrm{A}$ & 1207543 & J \\
\hline $10621 R$ & GGTAATITTCCGCTTGCGT & CGGTAATTITCCGCTTGCTC & GCACGGGCGCAGGCTCTTAT & 250 & $\begin{array}{l}\text { PET/ } \\
\text { NED }\end{array}$ & $\mathrm{C} / \mathrm{T}$ & 1207539 & J \\
\hline
\end{tabular}

First allele is the ancestral SNP state and has a VIC or NED dye; second allele is derived and has a FAM or PET dye. Universal tails were added to the 5 ' end of the allelic primers during primer synthesis. See Figures 1 and S1 for branch location of SNPs in phylogeny. SNP positions are given for B. melitensis 16 M genome and all are on chromosome I except assays 6214 and 2995 are on chromosome II. 
algorithm, simple sequence addition and TBR branch swapping [29]. We rooted the phylogeny with Brucella sp. 83/13 because of its basal position in the Brucella phylogeny for the isolates in our screening panel (unpubl. data). The 83/13 isolate came from an Australian rodent and data suggest that it is related to the traditional Brucella spp. [30] but likely diverged from the main/core Brucella. Using the phylogeny developed from the MIP assay to determine groups, we employed cladespecific SNPs using CUMA [31], following mismatch amplification concepts [32,33]. Briefly, the CUMA assay exploits mismatch amplification differences during PCR amplification that generate different length fragments that are allele (i.e. SNP) specific. The amplification primers have unique tails that can subsequently bind to fluorescently labeled universal-tailed primers. These unique tails are added to the 5 ' end of the allelic primers during primer synthesis. We used the following sequences and dyes for the universal tails: VICacacgcacttgacttgtcttc, FAM6-acccaactgaatagagagc, PETctgtccttacctcaatctc, NED-atcgactgtgttaggtcac. Assays were based on a range of amplicon sizes without overlap (range 101-259 bp) and two different dye combinations were used to visualize the fragments that discriminated each SNP state (VIC/FAM, NED/PET) (Table 1). We ran $10 \mathrm{~mL}$ reactions in either singleplex or multiplex with the following mastermix: $1 \mathrm{X}$ PCR buffer, $2 \mathrm{mM} \mathrm{MgCl}_{2}$, $0.2 \mathrm{mM}$ dNTPs (Invitrogen, Carlsbad, CA), $0.2 \mathrm{U}$ iTaq DNA Polymerase (Bio-Rad, Hercules, CA), and molecular grade water (GIBCO, Carlsbad, CA). Reactions were multiplexed in two different PCR reactions and fragments were run in a single capillary injection on an ABI 3100 Genetic Analyzer (Applied Biosystems, Foster City, CA). Thermocycler conditions were as follows: hot start of $95^{\circ} \mathrm{C}$ for $5 \mathrm{~min}$, followed by 40 cycles of $30 \mathrm{~s}$ at $95^{\circ} \mathrm{C}$, $30 \mathrm{~s}$ at $60^{\circ} \mathrm{C}$ and $30 \mathrm{~s}$ at $72^{\circ} \mathrm{C}$, with a final extension at $72^{\circ} \mathrm{C}$ for $5 \mathrm{~min}$. Melting temperatures for the primers ranged from 57.1 to $67.0^{\circ} \mathrm{C}$ (mean $61.7^{\circ} \mathrm{C}$ ).

SNPs used in the CUMA were randomly selected from the various options available on each branch, with fewer options possible with shorter branches. If development of the assay failed to produce effective primer pairs based on standard primer design parameters we simply selected a new SNP locus. Using the CUMA assays, we genotyped a diverse set of isolates $(n=340)$, which included all recognized biovars and type strains (except B. microti and B. suis biovars 3 and 5), against 17 SNP assays for 10 branches. For each sample we determined if the SNP allele for each locus was ancestral or derived on the corresponding branch and then verified where the sample was placed on the tree. When possible, we selected two SNPs from each of the major branches. We generated amplicons for the SNP regions in four PCR reactions for each of the two multiplex PCRs and then pooled the PCR product in one capillary injection. If the CUMA assay failed any locus in multiplex reactions, we reran that locus in singleplex, which generally allowed for determination of the SNP allele. Samples with singleplex failure largely appeared to be of poor DNA quality since there were typically failures across several different CUMA assays (Additional file 4: Table S2).

\section{Additional files}

\begin{abstract}
Additional file 1: Figure S1. Brucella phylogeny using maximum parsimony developed using 777 single nucleotide polymorphisms. Letters on branches refer to phylogenetic locations of CUMA assays developed in this work. Stars on branches represent phylogenetic locations of species or clade specific assays from Foster et al. 2008. In this figure we rooted with $B$. neotomae because it is the most basal taxon in the Brucella phylogeny for these taxa tested (unpubl. data).
\end{abstract}

Additional file 2: Table S3. List of Brucella DNA samples tested with CUMA. DNA samples came from the following institutions, Louisiana State University (LSU), California Department of Health Services (CDHS), U.S. Armed Forces Institute of Pathology (AFIP), Alaska Public Health Laboratory (APHL), Brigham Young University (BYU), U.S. Centers for Disease Control (CDC), USDA-National Animal Disease Center (NADC), and the Arizona Department of Health Services (ADHS). Samples with a species name in the branch column were genotyped as that species using assays in (Foster et al. 2008) but gave all ancestral SNP alleles in our assays. Assays for B. abortus in blue B. melitensis in pink, and B. suis/canis in green, which correspond to the branches in Figure 1. The 85 samples also run in the MIP assay have an asterisk, except for 3 samples not run on CUMA. Samples likely mislabeled, due to incorrect branch assignment based on species/biovar, are highlighted in yellow.

Additional file 3: Table S1. List of 28 whole genomes used for in silico comparisons to SNP alleles from MIP assay.

Additional file 4: Table S2. List of Brucella isolates used in 17 CUMA assays, including isolate name, species, and biovar when known or applicable and the SNP allele for each assay.

\section{Authors' Contributions}

JTF wrote the manuscript and performed data analysis; LBP carried out molecular genetic analysis and helped draft the manuscript; WDB, DMK, CAA, and CML performed molecular genetic analysis for MIPs and CUMA; SMBS, TP, and JBS carried out data analysis; FFR provided strains, data analysis and helped draft the manuscript; PK conceived of the study, participated in its design, and helped draft the manuscript. All authors read and approved the final manuscript.

\section{Acknowledgements}

We thank numerous contributors of DNA to our Brucella collection, including Brian Bell, Bryan Bellaire, Wally Buchholz, Robert Burgess, Barun De, Mike Dobson, Linda Getsinger, Ted Hadfield, and William Slanta. We thank Jim Schupp, Molly Matthews, and Jodi Beaudry for assistance with CUMA primer design and Ray Auerbach, Jolene Bowers, and Josh Colvin for help with data analysis and running samples. Recent whole genomes for comparisons were generated by the Broad Institute under the direction of David O'Callaghan, Adrian Whatmore, and Doyle Ward. Funding from the U.S. Department of Homeland Security (DHS) supported this work. Use of product or trade names does not constitute endorsement by the U.S. Government.

\section{Author details}

${ }^{1}$ Center for Microbial Genetics and Genomics, Northern Arizona University, Flagstaff, AZ 86011-4073, USA. ${ }^{2}$ Translational Genomics Research Institute, Flagstaff, AZ 86001, USA. ${ }^{3}$ Translational Genomics Research Institute, Phoenix, AZ 85004, USA. ${ }^{4}$ Idaho National Laboratory, Idaho Falls, ID 83415, USA.

Received: 8 December 2011 Accepted: 4 June 2012

Published: 19 June 2012 
References

1. Cloeckaert A, Vizcaino N: DNA polymorphism and taxonomy of Brucella species. In Brucella: Molecular and Cellular Biology. Edited by Lopez-Goni I, Moriyon I. Norfolk, UK: Horizon Bioscience; 2004:1-24.

2. Verger JM, Grimont F, Grimont PAD, Grayon M: Brucella, a monospecific genus as shown by deoxyribonucleic acid hybridization. Int J Syst Bacteriol 1985, 35:292-295.

3. Moreno E, Cloeckaert A, Moriyon I: Brucella evolution and taxonomy. Vet Microbiol 2002, 90(1-4):209-227.

4. Corbel MJ, Brinley-Morgan WJ: Genus Brucella Meyer and Shaw 1920 Baltimore, MD: Williams and Wilkins; 1984.

5. Osterman B, Moriyon I: International committee on systematics of prokaryotes: subcommittee on the taxonomy of Brucella. Int J Syst Evol Microbiol 2006, 56:1173-1175

6. Foster G, Osterman BS, Godfroid J, Jacques I, Cloeckaert A: Brucella ceti sp. nov. and Brucella pinnipedialis sp. nov. for Brucella strains with cetaceans and seals as their preferred hosts. Int J Syst Evol Microbiol 2007, 57(Pt 11):2688-2693.

7. Scholz HC, Hubalek Z, Sedlacek I, Vergnaud G, Tomaso H, Al Dahouk S, Melzer F, Kampfer P, Neubauer H, Cloeckaert A, et al: Brucella microti sp. nov., isolated from the common vole Microtus arvalis. Int J Syst Evol Microbiol 2008, 58(Pt 2):375-382.

8. Whatmore AM: Current understanding of the genetic diversity of Brucella, an expanding genus of zoonotic pathogens. Infect Genet Evol 2009, 9 (6):1168-1184

9. De BK, Stauffer L, Koylass MS, Sharp SE, Gee JE, Helsel LO, Steigerwalt AG, Vega R, Clark TA, Daneshvar Ml, et al: Novel Brucella strain (BO1) associated with a prosthetic breast implant infection. J Clin Microbio 2008, 46(1):43-49.

10. Scholz HC, Nockler K, Gollner C, Bahn P, Vergnaud G, Tomaso H, Al Dahouk S, Kampfer P, Cloeckaert A, Maquart M, et al: Brucella inopinata sp nov., isolated from a breast implant infection. Int J Syst Evol Microbiol 2010, 60:801-808.

11. Tiller R, Gee J, Lonsway D, Gribble S, Bell S, Jennison A, Bates J, Coulter C, Hoffmaster A, De B: Identification of an unusual Brucella strain (BO2) from a lung biopsy in a 52 year-old patient with chronic destructive pneumonia. BMC Microbiol 2010, 10(1):23

12. Halling SM, Peterson-Burch BD, Bricker BJ, Zuerner RL, Qing Z, Li LL, Kapur V, Alt DP, Olsen SC: Completion of the genome sequence of Brucella abortus and comparison to the highly similar genomes of Brucella melitensis and Brucella suis. J Bacteriol 2005, 187(8):2715-2726.

13. Paulsen IT, Seshadri R, Nelson KE, Eisen JA, Heidelberg JF, Read TD, Dodson RJ, Umayam L, Brinkac LM, Beanan MJ, et al: The Brucella suis genome reveals fundamental similarities between animal and plant pathogens and symbionts. Proceedings of the National Academy of Sciences USA 2002, 99(20):13148-13153.

14. Foster JT, Beckstrom-Sternberg SM, Pearson T, Beckstrom-Sternberg JS, Chain PSG, Roberto FF, Hnath J, Brettin T, Keim P: Whole genome-based phylogeny and divergence of the genus Brucella. J Bacteriol 2009, 191:2864-2870.

15. Hall $\mathrm{N}$ : Advanced sequencing technologies and their wider impact in microbiology. J Exp Biol 2007, 210(Pt 9):1518-1525.

16. Hardenbol $P$, Baner J, Jain M, Nilsson M, Namsaraev EA, Karlin-Neumann GA, Fakhrai-Rad H, Ronaghi M, Willis TD, Landegren U, et al: Multiplexed genotyping with sequence-tagged molecular inversion probes. Nat Biotechnol 2003, 21(6):673-678.

17. Keim P, Van Ert MN, Pearson T, Vogler AJ, Huynh LY, Wagner DM: Anthrax molecular epidemiology and forensics: using the appropriate marker for different evolutionary scales. Infect Genet Evol 2004, 4(3):205-213.

18. Foster JT, Okinaka RT, Svensson R, Shaw K, De BK, Robison RA, Probert WS, Kenefic $L$, Brown WD, Keim P: Real-time PCR assays of single-nucleotide polymorphisms defining the major Brucella clades. J Clin Microbiol 2008, 46:296-301.

19. Gopaul KK, Koylass MS, Smith CJ, Whatmore AM: Rapid identification of Brucella isolates to the species level by real time PCR based single nucleotide polymorphism (SNP) analysis. BMC Microbiol 2008, 8:86

20. Whatmore AM, Perrett LL, MacMillan AP: Characterisation of the genetic diversity of Brucella by multilocus sequencing. BMC Microbiol 2007, 7:34.

21. Pearson T, Okinaka RT, Foster JT, Keim P: Phylogenetic understanding of clonal populations in an era of whole genome sequencing. Infect Genet Evol 2009, 9(5):1010-1019.
22. Pearson T, Busch JD, Ravel J, Read TD, Rhoton SD, U'Ren JM, Simonson TS, Kachur SM, Leadem RR, Cardon ML, et al: Phylogenetic discovery bias in Bacillus anthracis using single-nucleotide polymorphisms from wholegenome sequencing. Proceedings of the National Academy of Sciences USA 2004, 101(37):13536-13541

23. Worobey M: Genomics: Anthrax and the art of war (against ascertainment bias). Heredity 2005, 94:459-460.

24. Audic S, Lescot M, Claverie JM, Cloeckaert A, Zygmunt MS: The genome sequence of Brucella pinnipedialis B2/94 sheds light on the evolutionary history of the genus Brucella. BMC Evol Biol 2011, 11:200.

25. DelVecchio VG, Kapatral V, Redkar RJ, Patra G, Mujer C, Los T, Ivanova N, Anderson I, Bhattacharyya A, Lykidis A, et al: The genome sequence of the facultative intracellular pathogen Brucella melitensis. Proceedings of the National Academy of Sciences USA 2002, 99(1):443-448.

26. Chain PSG, Comerci DJ, Tolmasky ME, Larimer FW, Malfatti SA, Vergez LM, Aguero F, Land ML, Ugalde RA, Garcia E: Whole-genome analyses of speciation events in pathogenic brucellae. Infect Immun 2005, 73(12):8353-8361.

27. Hardenbol P, Yu FL, Belmont J, MacKenzie J, Bruckner C, Brundage T, Boudreau A, Chow S, Eberle J, Erbilgin A, et al: Highly multiplexed molecular inversion probe genotyping: Over 10,000 targeted SNPs genotyped in a single tube assay. Genome Res 2005, 15(2):269-275.

28. Vogler AJ, Birdsell D, Price LB, Bowers JR, Beckstrom-Sternberg SM Auerbach RK, Beckstrom-Sternberg JS, Johansson A, Clare A, Buchhagen JL, et al: Phylogeography of Francisella tularensis: global expansion of a highly fit clone. J Bacterio/ 2009, 191(8):2474-2484

29. Swofford DL: PAUP*. Phylogenetic analysis using parsimony $*$ and other methods), version 4.0. Sunderland, MA: Sinauer Associates; 2002.

30. Tiller RV, Gee JE, Frace MA, Taylor TK, Setubal JC, Hoffmaster AR, De BK: Characterization of novel Brucella strains originating from wild native rodent species in North Queensland, Australia. Appl Environ Microbiol 2010, 76(17):5837-5845.

31. Price EP, Matthews MA, Beaudry JA, Allred JL, Schupp JM, Birdsell DN, Pearson T, Keim P: Cost-effective interrogation of single nucleotide polymorphisms using the mismatch amplification mutation assay and capillary electrophoresis. Electrophoresis 2010, 31(23-24):3881-3888.

32. Cha RS, Zarbl H, Keohavong P, Thilly WG: Mismatch amplification mutation assay (MAMA): application to the $\mathrm{c}-\mathrm{H}-\mathrm{ras}$ gene. PCR Methods and Application 1992, 2(1):14-20.

33. Li B, Kadura I, Fu D-J, Watson DE: Genotyping with TaqMAMA. Genomics 2004, 83(2):311-320

doi:10.1186/1471-2180-12-110

Cite this article as: Foster et al:: Genotyping of Brucella species using clade specific SNPs. BMC Microbiology 2012 12:110.

\section{Submit your next manuscript to BioMed Central and take full advantage of:}

- Convenient online submission

- Thorough peer review

- No space constraints or color figure charges

- Immediate publication on acceptance

- Inclusion in PubMed, CAS, Scopus and Google Scholar

- Research which is freely available for redistribution 